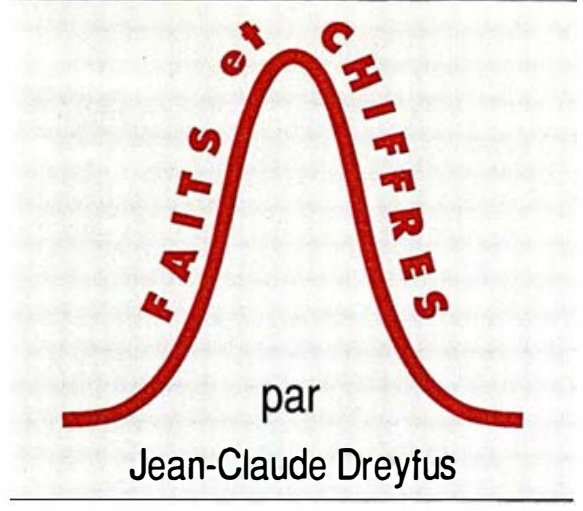

Jean-Claude Dreyfus

\title{
Tous les pays du monde
}

'étude sur "tous les pays du monde 1991 " provient des world population data sheet, établis, comme tous les deux ans, par le population reference bureau, et qui existe désormais en version française. Nous reproduisons dans ce numéro huit indicateurs, dont les six premiers

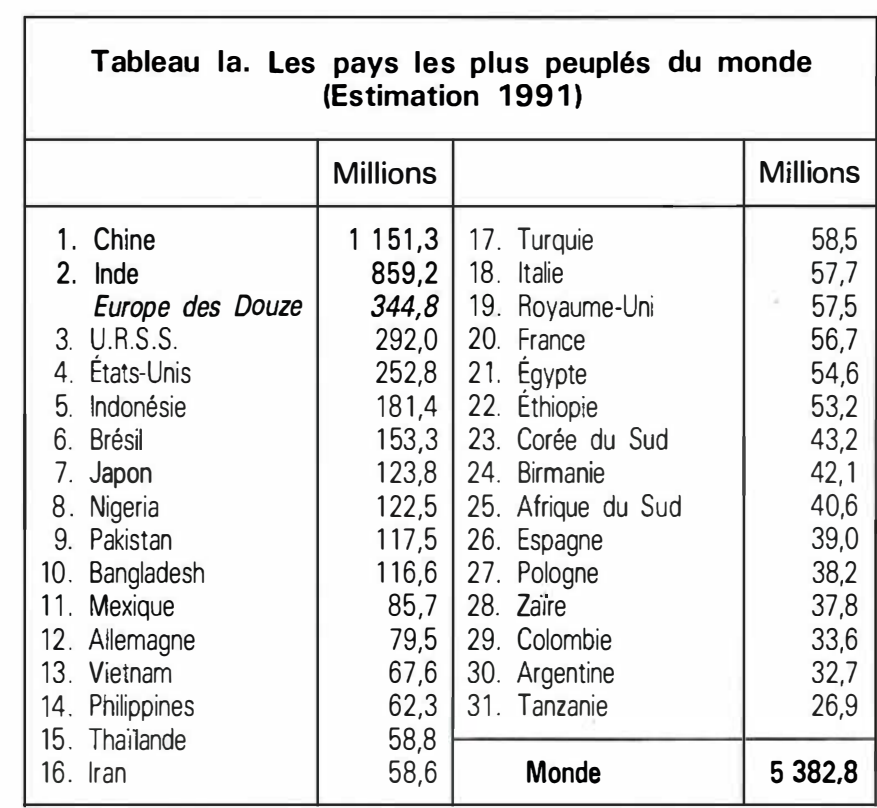

Tableau Ib. Naissances annuelles

\begin{tabular}{|c|c|c|c|}
\hline \multicolumn{4}{|c|}{ Tableau lb. Naissances annuelles } \\
\hline & Millions & & Millions \\
\hline 1. Inde & 26,64 & 17. Zaïre & 1,74 \\
\hline 2. Chine & 24,18 & 18. Afrique du Sud & 1,42 \\
\hline 3. Nigeria & 5,39 & 19. Birmanie & 1,35 \\
\hline 4. U.R.S.S. & 5,26 & 20. Tanzanie & 1,35 \\
\hline 5. Pakistan & 5,05 & 21. Japon & 1,24 \\
\hline 6. Indonésie & 4,54 & 22. Thailande & 1,18 \\
\hline 7. Bangladesh & 4,31 & 23. Kenya & 1,16 \\
\hline 8. États-Unis & 4,30 & 24. Soudan & 1,14 \\
\hline 9. Brésil & 4,14 & 25. Ouganda & 0,97 \\
\hline \multirow{7}{*}{$\begin{array}{l}\text { Europe des Douze } \\
\text { 10. Éthiopie } \\
\text { 11. Mexique } \\
\text { 12. Iran } \\
\text { 13. Vietnam } \\
\text { 14. Égypte } \\
\text { 15. Philippines } \\
\text { 16. Turquie }\end{array}$} & 4,14 & 26. Algérie & 0,91 \\
\hline & 2,61 & 27. Maroc & 0,89 \\
\hline & 2,49 & 28. Allemagne & 0,87 \\
\hline & 2,40 & 29. Colombie & 0,87 \\
\hline & 2,16 & 30. Népal & 0,82 \\
\hline & 2,07 & 31. Royaume-Uni & 0,81 \\
\hline & 1,76 & Monde & 143,7 \\
\hline
\end{tabular}

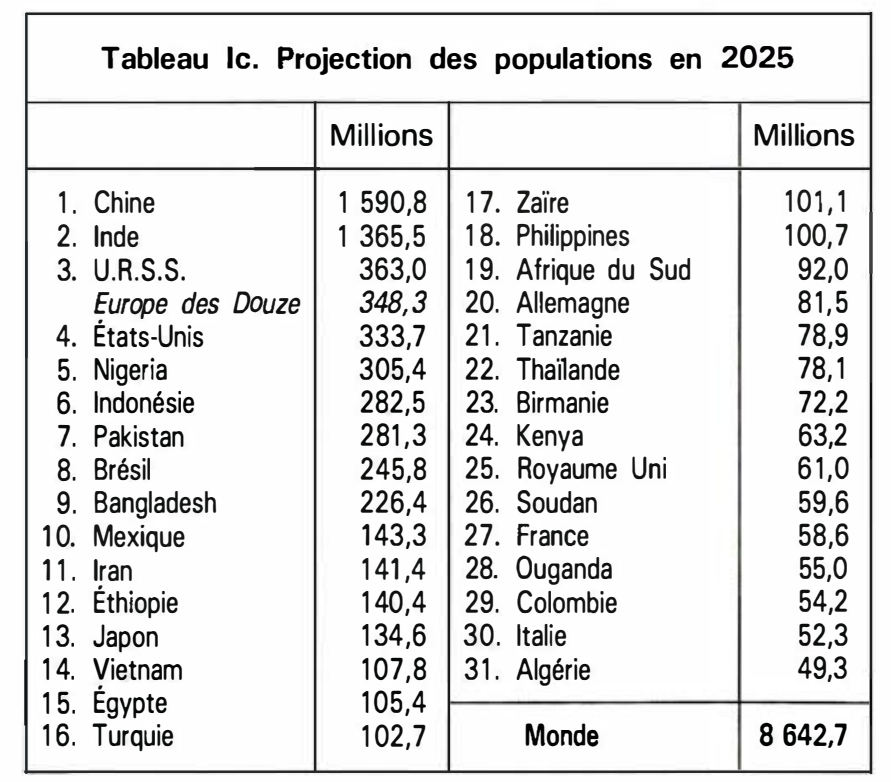

Source: Lévy ML, Population et Sociétés INED (Institut National d'Études Démographiques), juillet-août 1991, nº 259. correspondent, dans le même ordre, à ceux qu'a publiés $\mathrm{m} / \mathrm{s}$ en $1986\left(n^{\circ} 2\right.$, vol. 2, p. 108-9), afin de permettre les comparaisons. Allemagne et Yemen figurent sous forme réunifiée. En six ans la population mondiale a augmenté d'environ $10 \%$. Les projections (sur 2020 en 1985, sur 2025 en 1991) restent très voisines. On peut citer toutefois pour ces dernières une baisse des estimations pour les pays du Maghreb, et une hausse notable pour l'Allemagne, due, dans ce dernier cas, non à une remontée de la natalité, mais à une très forte immigration 


\begin{tabular}{|l|l|l|l|}
\hline \multicolumn{3}{|c|}{ Tableau Ila. Taux de natalité (pour 1 000 habitants) } \\
\hline Gaza & 54 & France & 14 \\
Malawi & 52 & Yougoslavie & 14 \\
Ouganda & 52 & Finlande & 13 \\
Mali & 51 & Pays-Bas & 13 \\
Niger & 51 & Bulgarie & 13 \\
Rwanda & 51 & Tchécoslovaquie & 13 \\
Zambie & 51 & Hong Kong & 12 \\
Yémen & 51 & Danemark & 12 \\
Burkina-Faso & 50 & Autriche & 12 \\
Côte-d'ivoire & 50 & Belgique & 12 \\
Togo & 50 & Luxembourg & 12 \\
Țanzanie & 50 & Suisse & 12 \\
Éthiopie & 49 & Hongrie & 12 \\
\cline { 1 - 2 } Monde & 27 & Portugal & 12 \\
\cline { 1 - 2 } Norvège & 14 & Allemagne & 11 \\
Royaume-Uni & 14 & Japon & 11 \\
Suède & 14 & Grèce & 10 \\
& & & 10 \\
\hline
\end{tabular}

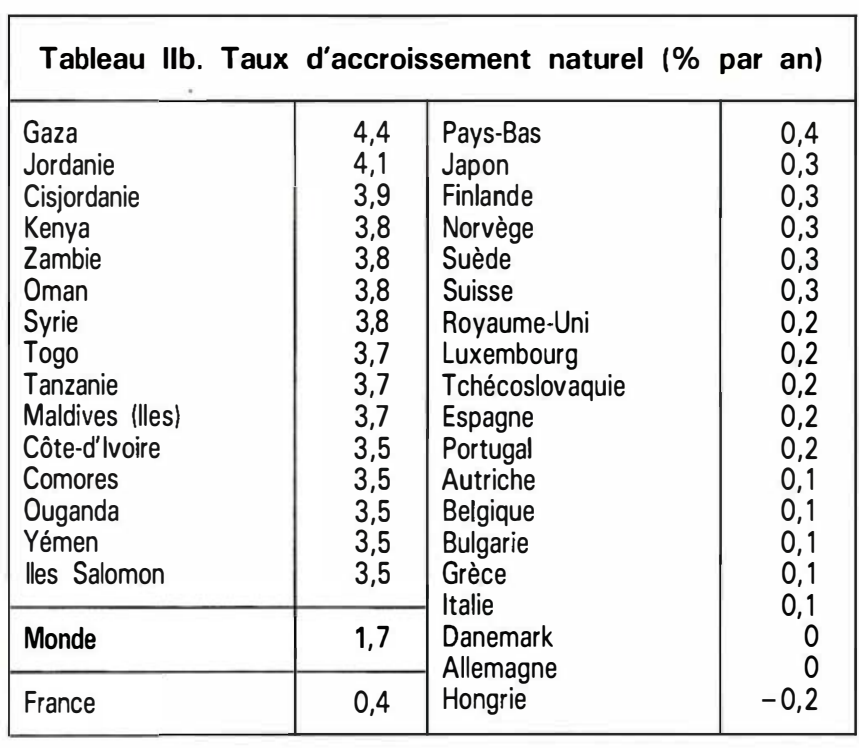

Tableau Ilc. Indice synthétique de fécondité (Enfants par femme)

\begin{tabular}{|l|r|l|r|}
\hline Rwanda & 8,1 & France & 1,8 \\
Malawi & 7,7 & Hongrie & 1,8 \\
Côte-d'lvoire & 7,4 & Taïwan & 1,7 \\
Ouganda & 7,4 & Canada & 1,7 \\
Yémen & 7,4 & Finlande & 1,7 \\
Burkina-Faso & 7,2 & Corée du Sud & 1,6 \\
Togo & 7,2 & Danemark & 1,6 \\
Zambie & 7,2 & Belgique & 1,6 \\
Arabie Saoudite & 7,2 & Pays-Bas & 1,6 \\
Bénin & 7,1 & Suisse & 1,6 \\
Mali & 7,1 & Japon & 1,5 \\
Niger & 7,1 & Allemagne & 1,5 \\
Tanzanie & 7,1 & Luxembourg & 1,5 \\
Jordanie & 7,1 & Grèce & 1,5 \\
Afghanistan & 7,1 & Portugal & 1,5 \\
Burundi & 7 & Autriche & 1,4 \\
\cline { 1 - 1 } Monde & \multirow{2}{*}{$\mathbf{3 , 4}$} & Espagne & 1,3 \\
& & Italie & 1,3 \\
& & Hong Kong & 1,2 \\
\hline
\end{tabular}

\begin{tabular}{|c|c|c|c|}
\hline \multicolumn{4}{|c|}{ Tableau Illa. Décès d'enfants de moins d'un an } \\
\hline & Millions & & Millions \\
\hline \multirow{16}{*}{$\begin{array}{l}\text { 1. Inde } \\
\text { 2. Chine } \\
\text { 3. Nigeria } \\
\text { 4. Pakistan } \\
\text { 5. Bangladesh } \\
\text { 6. Ethiopie } \\
\text { 7. Indonésie } \\
\text { 8. Brésil } \\
\text { 9. Égypte } \\
\text { 10. Afganistan } \\
\text { 11. Zaïre } \\
\text { 12. Tanzanie } \\
\text { 13. Birmanie } \\
\text { 14. U.R.S.S. } \\
\text { 15. Soudan } \\
\text { 16. Philippines } \\
\text { 17. Turquie }\end{array}$} & \multirow{16}{*}{$\begin{array}{l}2,42 \\
0,80 \\
0,64 \\
0,57 \\
0,52 \\
0,34 \\
0,33 \\
0,26 \\
0,15 \\
0,15 \\
0,14 \\
0,14 \\
0,13 \\
0,12 \\
0,12 \\
0,11 \\
0,11\end{array}$} & 18. Mexique & 0,11 \\
\hline & & 19. Ouganda & 0,10 \\
\hline & & 20. Iran & 0,10 \\
\hline & & 21. Mozambique & 0,10 \\
\hline & & 22. Vietnam & 0,10 \\
\hline & & 23. Népal & 0,09 \\
\hline & & 24. Afrique du Sud & 0,07 \\
\hline & & 25. Kenya & 0,07 \\
\hline & & 26. Algérie & 0,07 \\
\hline & & 27. Maroc & 0,07 \\
\hline & & 28. Malawi & 0,07 \\
\hline & & 29. Madagascar & 0,06 \\
\hline & & 30. Yémen & 0,06 \\
\hline & & 31. Côte-d'lvoire & 0,06 \\
\hline & & Europe des Douze & \\
\hline & & Monde & 9.71 \\
\hline
\end{tabular}

Tableau IIIb. P.I.B. 1989 (Milliards de dollars)

\begin{tabular}{|l|r|l|r|}
\hline 1. États-Unis & 5132,0 & 17. Mexique & 197,8 \\
Europe des Douze & 4848,0 & 18. Suède & 189,3 \\
2. Japon & 2770,7 & 19. Suisse & 174,6 \\
3. U.R.S.S. & 2052,8 & 20. Iran & 168,9 \\
4. Allemagne & 1189,1 & 21. Belgique & 152,9 \\
5. France & 955,2 & 22. Autriche & 126,3 \\
6. Italie & 864,3 & 23. Tchécoslovaquie & 118,5 \\
7. Royaume-Uni & 839,1 & 24. Finlande & 115,5 \\
8. Chine & 660,1 & 25. Roumanie & 112,4 \\
9. Canada & 542,8 & 26. Danemark & 104,4 \\
10. Espagne & 379,2 & 27. Taïwan & 96,4 \\
11. Brésil & 370,6 & 28. Norvège & 90,8 \\
12. Australie & 290,3 & 29. Afrique du Sud & 89,4 \\
13. Inde & 267,4 & 30. Indonésie & 88,6 \\
14. Pays-Bas & 223,8 & 31 . Égypte & 84,9 \\
15. Corée du Sud & 204,9 & \multicolumn{2}{|c|}{ Monde } \\
16. Pologne & 198,0 & & 20458,0 \\
\hline
\end{tabular}

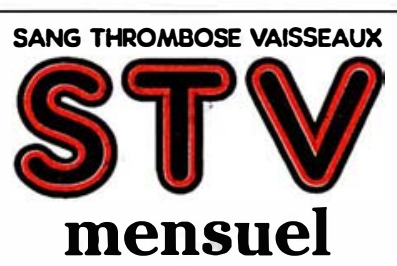

(10 numéros par an)

\section{Vient de paraître}

Vol. 3, n 7 - septembre 1991

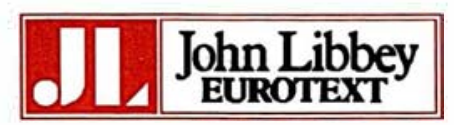

6, rue Blanche, 92120 Montrouge, France Tél. : 47.35.85.52 - Fax : 46.57.10.09 